\title{
Observation of the Gap and Kinetic Energy in a Correlated Insulator
}

\author{
G. A. Thomas, D. H. Rapkine, S. A. Carter, and A. J. Millis \\ AT\&T Bell Laboratories, Murray Hill, New Jersey 07974-0636 \\ T. F. Rosenbaum \\ Physics Department and the James Franck Institute, The University of Chicago, Chicago, Illinois 60637 \\ P. Metcalf and J. M. Honig \\ Department of Chemistry, Purdue University, West Lafayette, Indiana 47907
}

(Received 28 April 1994)

\begin{abstract}
Fundamental energies are determined optically for an ensemble of correlated electrons in an antiferromagnetic insulator, $\mathrm{V}_{2} \mathrm{O}_{3}$. The observed variation of the energy gap and the kinetic energy are compared quantitatively to some approximate solutions of the Hubbard Hamiltonian.
\end{abstract}

PACS numbers: 71.27. $+\mathrm{a}, 71.30 .+\mathrm{h}, 78.30 . \mathrm{Hv}$

The approach to the metal-insulator transition by a collection of correlated electrons has been a subject of considerable interest since Mott's classic paper [1] in 1949. In the class of systems where the electrons attempt to form a half-filled band, but remain insulating, Mott [1] and Hubbard [2] have suggested that the band could have been split by the Coulomb repulsion among the electrons. Alternatively, Slater [3] suggested that antiferromagnetic interactions alone could open an energy gap to produce an insulator. These authors have argued that these general considerations apply in some form to a very wide class of condensed matter systems. Recently, new, approximate solutions [4-7] of the Hubbard model containing both these ideas have been studied extensively. The motivation of our experiments has been to compare these new calculations with the optical spectra of a model system of correlated electrons.

We have chosen $\mathrm{V}_{2} \mathrm{O}_{3}$ because there are some indications $[8,9]$ that it may be approximated by a Hubbard model. Band-structure calculations [10] show that a range of V-V transitions (from predominantly V initial states to predominantly $\mathrm{V}$ final states) dominates the spectrum up to about $3.5 \mathrm{eV}$ and a range of $\mathrm{V}-\mathrm{O}$ transitions occurs only at higher $E$. We find that its optical conductivity is in good agreement with the band structure. Anderson [11] has suggested that the Hubbard model applies with an effective (rather than a bare) Coulomb interaction energy. Brinkman and Rice [12], for example, have argued further that the model applies with magnetic correlations relatively unimportant in the metallic state.

Another motivation for our choice of $\mathrm{V}_{2} \mathrm{O}_{3}$ was that carefully characterized crystals $[9,13,14]$ were available. We have been able to measure the optical energy gap, which, to our knowledge, had not been measured previously, partly because of the improved materials. Previously, an energy gap had been inferred from various measurements, such as the temperature dependence of the resistivity $[8,13,14]$. However, the only far-infrared optical measurements [15] showed a reduction in the conduc- tivity at low $E$ but no energy gap. A similar reduction in the density of states has been observed in photoemission spectra [16], also with no spectral region with negligible signal intensity.

We have made measurements with energy resolution less than $1 \mathrm{meV}$ on crystals which we found to be very difficult to anneal, polish, and etch, with the additional difficulty that they crack on passing through the metalinsulator transition that occurs on cooling from room temperature. Based on optical and dc transport characterization of over 10 crystals, we have developed annealing and surface preparation procedures which yield reproducible spectra [17]. We have made reflectivity, $R$, measurements from the far-infrared to $E=3.5 \mathrm{eV}$ (a frequency, $\omega$, about $27000 \mathrm{~cm}^{-1}$ ) and used the measurements of Shin et al. [18] from 3.5 to $25 \mathrm{eV}$ in our Kramers-Kronig transformations to obtain the optical conductivity, $\sigma$.

The main part of Fig. 1 shows $\sigma$ at low $E$ for two crystals of $\mathrm{V}_{2} \mathrm{O}_{3}$ in their low-temperature insulating phase. The open circles are for a nominally stoichiometric crystal with $T_{c}=154 \pm 1 \mathrm{~K}$, measured at $70 \mathrm{~K}$. The solid circles are for a sample, measured at $T=10 \mathrm{~K}$, that was annealed differently to introduce $\mathrm{V}$ vacancies and a reduced $T_{c}=50 \pm 1 \mathrm{~K}$. We found that no substantial changes occurred at lower $T$, so that our results are representative of the zero- $T$ limit. The effect of this annealing is qualitatively similar to an effective pressure [14]. It differs from doping a rigid band without disorder (often discussed in the theoretical literature [4,5]) which can introduce conducting states into a filled band, and it also differs from disordered doping which primarily introduces impurity states into the gap [1]. We see negligible absorption (except that due to phonons) in the energy gap, and our $\sigma$ extrapolated to $E=0$ agrees with the (essentially zero) measured dc conductivity, $\sigma_{\mathrm{dc}}$, of similarly prepared samples.

Qualitatively, the spectra show an energy gap, $2 \Delta$, with a "soft" edge and a broad peak at higher energy. This 


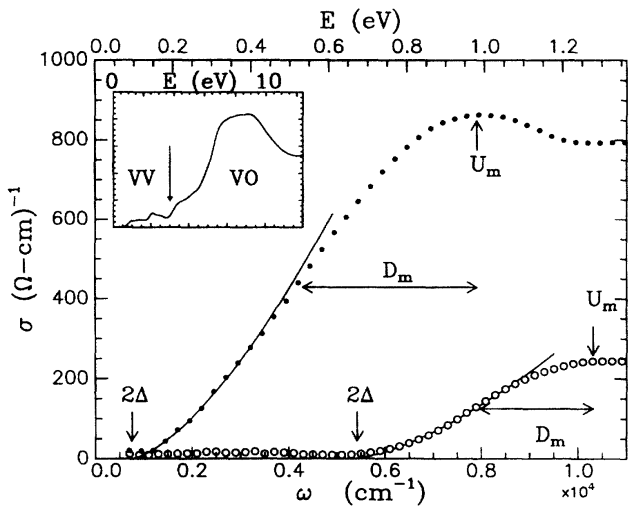

FIG. 1. Optical conductivity $\sigma$ as a function of photon frequency $\omega$ (lower scale) and energy $E$ (upper scale), for two crystals of $\mathrm{V}_{2} \mathrm{O}_{3}$ with metal-insulator transition temperatures, $T_{c}=50 \mathrm{~K}$ (solid points) and $154 \mathrm{~K}$ (open circles). The energy gap $2 \Delta$, half-width $D_{m}$, and peak energy $U_{m}$ are indicated. The solid lines are fits by Eq. (1). The inset shows a wider region of the spectrum for the sample with $T_{c}=154 \mathrm{~K}$ with $\sigma$ over a scale 0 to 5000 and $E$ from 0 to $12.5 \mathrm{eV}$.

type of spectrum resembles that predicted by various models $[5,7,19,20]$ with no intersite spin correlations. This behavior differs from our expectation for a Slater antiferromagnet [3], where we would expect a $E^{-1 / 2}$ divergence in the density of states at the band edge which would produce a $\sigma$ rising sharply as $E^{1 / 2}$. We expect that the peak in $\sigma$ arises from a superposition of the states in the (filled) lower Hubbard band and the (empty) upper Hubbard band. If these single-particle densities of states rise as $E^{1 / 2}$ near the band edges, we argue that $\sigma$ will rise as $E^{3 / 2}$ just above $2 \Delta$. We then determine the values of $2 \Delta$ using a fit with this form (solid lines, Fig. 1):

$$
\sigma=\sigma_{0}(E-2 \Delta)^{3 / 2}
$$

where $\sigma_{0}$ is a constant related to the average $\mathrm{ki}$ netic energy. The constants are $\left[T_{c}, \sigma_{0}, 2 \Delta\right]=$ $\left[154 \mathrm{~K}, 9.71 \times 10^{-4}(\Omega \mathrm{cm})^{-1}, \quad 0.66 \pm 0.05 \mathrm{eV}\right] \quad$ and $\left[50 \mathrm{~K}, 2.27 \times 10^{-3}(\Omega \mathrm{cm})^{-1}, 0.09 \pm 0.05 \mathrm{eV}\right]$.

The definition of the effective Coulomb repulsion energy $U$ is the energy difference between the peaks of the lower and upper Hubbard bands in the single-particle density of states. For large $U$, the absolute $E$ of the maximum in $\sigma, U_{m}$ (see arrow in Fig. 1), corresponds $[7,19,20]$ to $U$. Our values are $\left[T_{c}, U_{m}\right]=[154 \mathrm{~K}, 1.27 \pm$ $0.05 \mathrm{eV}]$ and [ $50 \mathrm{~K}, 0.98 \pm 0.05 \mathrm{eV}]$.

The hopping energy $t$ which appears in the usual form of the Hubbard Hamiltonian, is proportional to the bandwidth $D$ through the number of nearest-neighbor hopping sites. We define the full width of the single particle bands to be $2 D$, so the half width at half maximum of the peak in the measured $\sigma, D_{m}$, corresponds to $D$. The uncertainty is similar to that in $U_{m}$, giving values $\left[T_{c}, D_{m}\right]=[154 \mathrm{~K}, 0.31 \pm 0.05 \mathrm{eV}]$ and $[50 \mathrm{~K}, 0.47 \pm$
$0.05 \mathrm{eV}$. These narrow bandwidths are comparable to band-structure calculations for individual bands in the $d$ to $d$ manifold [10], and the increase in $D_{m}$ in the lower $T_{c}$ sample is consistent with the idea of an increasing effective pressure that increases the overlap of the atomic wave functions.

We plot the optical measurements of the energy gap (normalized to $D_{m}$ ) as a function of the characteristic energy ratio, $U_{m} / D_{m}$ (solid points, bottom scale), in Fig. 2. For additional measurements of the gap, we have used dc results from samples with different stoichiometries [13] and applied pressures [14]. These measurements determined the excitation energy, $\Delta_{\mathrm{dc}}$, from the slope of $\ln \left(\sigma_{\mathrm{dc}}\right)$ versus $1 / T$. We find that, for samples with $T_{c}=154 \pm 4 \mathrm{~K}, 2 \Delta_{\mathrm{dc}}$ is about $2 \Delta / 1.7$, and we interpret this smaller transport value as indicating that the chemical potential is pinned at a weak impurity level (not apparent on the scale of Fig. 1), rather than floating at midgap. Therefore, we have scaled the values of $2 \Delta_{\mathrm{dc}}$ by 1.7. We have used the measured values of $T_{c}$ to estimate the values of $U_{m}$ and $D_{m}$ for the dc data by interpolating between the optical values. We assume $U_{m} / D_{m}=(U / D)_{0}\left(1+\left[T_{c} / T_{0}\right]^{3}\right)$ and find $T_{0}=152 \mathrm{~K}$ and $(U / D)_{0}=2.01$ by fitting to the optical data. We have chosen this $T_{c}^{3}$ functional form because we can fit the dc data to $2 \Delta_{\mathrm{dc}}=2 \Delta_{0}\left(T_{c} / T_{0}\right)^{3}$, with $2 \Delta_{0}=0.4 \mathrm{eV}$. This $T^{3}$ variation may be due to the interplay between the ground state Coulomb energy and the lattice excitation energy at increasing $T$. We use a similar scaling to interpolate values of $D_{m}$ for the dc data, $1 / D_{m}=1 / D_{0}\left(1+\left[T_{c} / T_{D}\right]^{3}\right)$, with $D_{0}=0.48 \mathrm{eV}$

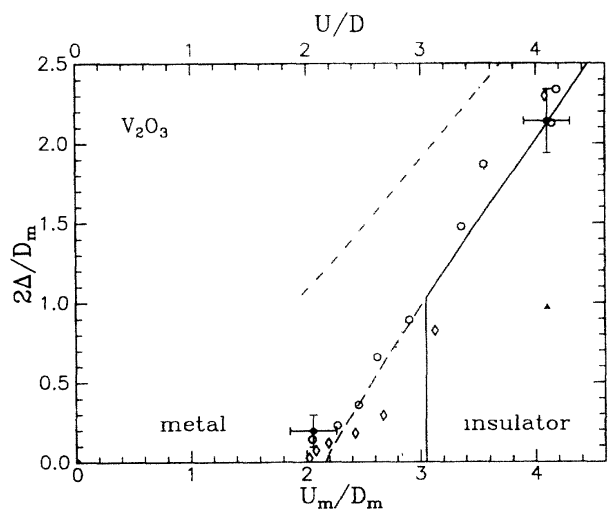

FIG. 2. Energy gap $2 \Delta$, normalized to the experimental bandwidth $D_{m}$, as a function of the normalized experimental band energy $U_{m}$ (bottom scale), for the optical measurements (solid circles), and for the dc measurements with annealing (open circles) and with pressure (open diamonds). The calculations of $2 \Delta / D$ using the dynamical mean-field model [20] are plotted against the relative Coulomb repulsive energy, $U / D$ (top scale), for the Slater limit (dash-dotted line) and the stable (solid line) and metastable (dashed) branches of the Hubbard limit. A value calculated for $\mathrm{V}_{2} \mathrm{O}_{3}$ using the Hartree-Fock approximation [6] is shown as the solid triangle. A slave-boson calculation [7] in the Hubbard limit is also shown (dotted line). 
and $T_{D}=188 \mathrm{~K}$. This procedure yields the open points in Fig. 2. The annealed series of samples [13] and the sample at a series of pressures [14] yield similar results. A linear extrapolation of all the data suggests a continuous gap closing at $\left(U_{m} / D_{m}\right)_{c}=1.95 \pm 0.2$.

We compare these measurements with theoretical predictions for $2 \Delta / D$ in Fig. 2 by plotting the theoretical $U / D$ on the upper scale, with the assumption (accurate for large $U / D$ that $U / D=U_{m} / D_{m}$. We consider four types of theoretical approaches: (1) perturbation [4,21] about the exactly solvable limits, $U / D=0$ and $U / D=\infty$, (2) Hartree-Fock mean-field [6] (3) slave-boson mean-field [7], and (4) dynamical mean-field [5]. The first three can be applied directly to models in three spatial dimensions. The Hartree-Fock approach has been applied to a model incorporating many of the aspects of the crystal structure of $\mathrm{V}_{2} \mathrm{O}_{3}$. The fourth is calculated for infinite dimensions, but may give a good approximation for three dimensions in the insulator. It also has the advantage of validity for arbitrary $U / D$ and inclusion of correlations not easy to incorporate in other approaches. Within each theoretical approach there are two simple limits for treating the magnetism. One limit assumes that the intersite spin correlations are zero, so the gap arises simply from $U$, which we shall refer to as the Hubbard limit. The second assumes simple, two-sublattice, antiferromagnetic order, so a large, additional contribution to the gap arises from the magnetism (the Slater limit).

Relatively large gaps are obtained in the Slater limit as shown by the dash-dotted curve for the dynamical meanfield theory [20]. The Hartree-Fock calculation [6] gives a lower result, $2 \Delta=0.3 \mathrm{eV}$, as shown by the solid triangle plotted using $U_{m}$ and $D_{m}$ for the sample with $T_{c}=154 \mathrm{~K}$. (Because of the realistic structure used here, the treatment of the magnetism is between the two magnetic limits.) Smaller values of the gap also come from consideration of the Hubbard limit as illustrated by the dotted line from a slave-boson approximation on a simple cubic lattice [7], given by $2 \Delta / D_{m}=\left(U_{m} / D_{m}-2.6\right)^{1 / 2}$. The solid line is from a calculation of the dynamical mean-field model in the Hubbard limit [20]. In this limit, a metalinsulator transition occurs at $U / D=U_{c 2} / D=3.05$, with the formation of a band of extended quasiparticle states in the middle of a gap. However, this metallic state is only about $0.1 \%$ lower in energy than an insulating solution in which the gap closes continuously at $U_{c 1} / D=2.15$ as shown by the dashed line. It seems likely $[5,20]$ that a small magnetic contribution to the energy would stabilize the insulating solution without changing the values of $2 \Delta$ substantially. Such a small antiferromagnetic contribution could arise because the spin structure of $\mathrm{V}_{2} \mathrm{O}_{3}$ shows substantial, but incomplete, frustration [22] due to a mixture of ferromagnetic and antiferromagnetic, nearestneighbor interactions.

The data of Fig. 1 permit an additional test of the theory. Qualitatively, there is a dramatic increase in the spectral weight (area under the curve) with decreasing $U_{m} / D_{m}$. This weight provides a measure of the average electronic kinetic energy, $\langle\hat{T}\rangle$. Kohn and subsequent workers $[23,24,25]$ have shown that $\langle\hat{T}\rangle /\langle\hat{T}\rangle_{0}=\omega_{P}^{2} / \omega_{P 0}^{2}$. Here, we have normalized $\langle\hat{T}\rangle$ and $\omega_{P}^{2}$ to their values for the same bandwidth, but for $U=0,\langle\hat{T}\rangle_{0}$, and $\omega_{P 0}^{2}$. Using the $f$-sum rule $[23,25]$, we determine $\omega_{P}^{2}$ from an integral over $\sigma$. Since we find additional bands at higher $E$ in $\mathrm{V}_{2} \mathrm{O}_{3}$, we have assumed symmetric bands and doubled the integral over the lower $E$ half of the conductivity peak:

$$
\omega_{P}^{2}=16 \int_{0}^{U_{m}} \sigma d E
$$

For the two data sets shown in Fig. 1, we have $\left[T_{c}, \omega_{P}^{2}\right]=$ $\left[154 \mathrm{~K}, 0.23 \pm 0.04 \mathrm{eV}^{2}\right]$ and $\left[50 \mathrm{~K}, 1.2 \pm 0.04 \mathrm{eV}^{2}\right]$.

We evaluate $\omega_{P 0}^{2}$, following the calculations of Millis and Coppersmith [25], using a half-filled cubic lattice, the atomic kinetic energy, and the measured bandwidth and find

$$
\omega_{P 0}^{2}=1.4 D_{m} e^{2} / d_{\mathrm{Vv}},
$$

where $d_{\mathrm{vv}}$ is the average vanadium-vanadium distance in $\mathrm{V}_{2} \mathrm{O}_{3}, 3.06 \AA$. For the two crystals, we then have $\left[T_{c},\langle\hat{T}\rangle /\langle\hat{T}\rangle_{0}\right]=[154 \mathrm{~K}, 0.11 \pm 0.05 \mathrm{eV}]$ and $[50 \mathrm{~K}$, $0.39 \pm 0.05 \mathrm{eV}]$.

Using these determinations, we can compare $\langle\hat{T}\rangle /\langle\hat{T}\rangle_{0}$ from experiment with that from the dynamical meanfield model in Fig. 3 with no adjustable parameters. The calculated variation [20] for the (partly metastable) insulating branch of the Hubbard limit is shown as the solid and dashed curves, and the data are shown as solid points. The comparable calculation for the metallic state

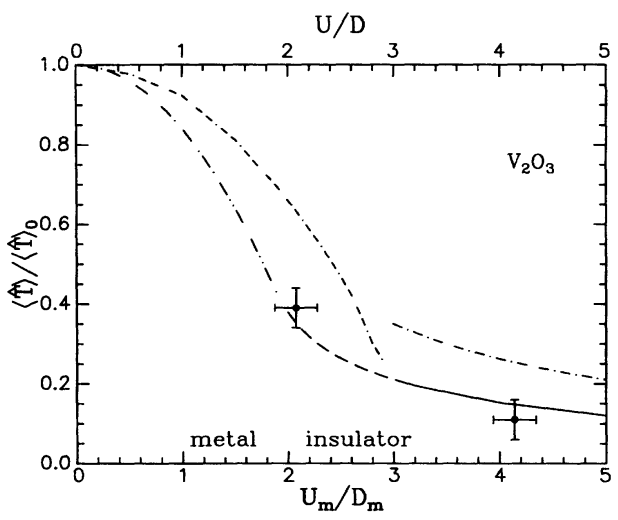

FIG. 3. Average kinetic energy, $\langle\hat{T}\rangle$, normalized to its value for $U=0,\langle\hat{T}\rangle_{0}$, as a function of the same variables as Fig. 2 . The solid points are the optical measurements [evaluated using Eq. (2) and normalized using Eq. (3)]. The dynamical meanfield theory [20] is plotted in the Hubbard limit for its insulating stable branch (solid line) and metastable branch (dashed), and in the metallic state (dash-dash-dotted). Perturbative calculations are plotted for large $U / D$ in the Slater limit (dash-dotted) and for small $U / D$ (dash-dot-dotted). 
is similar to the result of Brinkman and Rice [12] and is shown by the dash-dash-dotted line. The Hubbard model can also be evaluated for small $U / D$ using perturbation theory [4] and extrapolated to the intermediate $U / D$ range of interest here. For small $U / D$ in infinite dimensions [4], the result is similar to the dash-dash-dotted line, and is given by $\langle\hat{T}\rangle /\langle\hat{T}\rangle_{0}=1-0.098(U / D)^{2}$ (not shown in the figure). In three dimensions [4,25], we find $\langle\hat{T}\rangle /\langle\hat{T}\rangle_{0}=1-0.16(U / D)^{2}$, which is shown as the dashdot-dotted curve. Similarly, for the large $U / D$ limit in three dimensions [21,25], we find $\langle\hat{T}\rangle /\langle\hat{T}\rangle_{0}=1.05 U / D$ (the dot-dashed curve) for the Slater limit and a result $1 / 2$ as large (near the solid curve, but not shown) for the Hubbard limit. All the curves show a suppression of the average kinetic energy (in addition to that expected from the bandwidth change) with increasing $U / D$.

We conclude that, although we have measured a correlated antiferromagnet, we have not observed the large energy gaps and large kinetic energies expected in the Slater limit of approximate solutions to the Hubbard Hamiltonian. Instead, perhaps as a result of spin frustration, a partly metastable solution near the Hubbard limit is closest to our data. We consider this agreement to indicate that the dynamical mean-field model is quantitatively successful in describing the apparently continuous closing of the energy gap and the accompanying rise of the kinetic energy in insulating $\mathrm{V}_{2} \mathrm{O}_{3}$.

We wish to acknowledge helpful discussions with E. Abrahams, S. Coppersmith, L.F. Mattheiss, T. M. Rice, M. J. Rozenberg, D. J. Scalapino, D. Vollhardt, and particularly G. Kotliar. The work at the University of Chicago was supported by NSF DMR9204820; at Purdue University by MISCON on DOE Grant No. DE-FG02-90ER45427.

[1] N.F. Mott, Philos. Mag. 6, 287 (1961); The MetalInsulator Transition (Taylor and Francis, London, 1974).

[2] J. Hubbard, Proc. R. Soc. London A 276, 238 (1963); ibid. 281, 401 (1964).

[3] J. C. Slater, Phys. Rev. 82, 538 (1951).

[4] For a review, see D. Vollhardt, in Correlated Systems, edited by V. J. Emery (World Scientific, Singapore, 1993), p. 57. Also, W. Metzner and D. Vollhardt, Phys. Rev. Lett. 62, 324 (1989); Phys. Rev. B 39, 4462 (1989); M. Kollar and D. Vollhardt (private communication).

[5] For a review, see G. Kotliar, in Strongly Correlated Materials, edited by K.S. Bedell et al. (Addison-Wesley, New York, 1994). See also, A. Georges and G. Kotliar, Phys. Rev. B 45, 6497 (1992); A. Georges and W. Krauth, Phys. Rev. Lett. 69, 1240 (1992); Phys. Rev. B 48, 7167 (1993); M. J. Rozenberg, X.Y. Zhang, and G. Kotliar, Phys. Rev. Lett. 69, 1236 (1992); ibid. 70, 1666 (1993); Phys. Rev. B 49, 10 181(1994).
[6] C. Castellani, C. R. Natoli, and J. Ranninger, Phys. Rev. B 18, 4967 (1978).

[7] R. Raimondi and C. Castellani, Phys. Rev. B 48, 11453 (1993); (private communication).

[8] See, for example, D. B. McWhan, A. Menth, J.P. Remeika, W.F. Brinkman, and T. M. Rice, Phys. Rev. B 7, 1920 (1973), and references therein.

[9] J. M. Honig and L. L. Van Zandt, Annu. Rev. Mat. Sci. 5, 225 (1975).

[10] L.F. Mattheiss (private communication); J.B. Goodenough, in Proceedings of the 10th International Conference on Physics of Semiconductors, edited by S.P. Keller, J.C. Hensel, and F. Stern (U.S. AEC, Oak Ridge, TN, 1970), p. 304; J. Ashkenazi and T. Chuchem, Philos. Mag. 32, 763 (1965).

[11] P. W. Anderson, in Frontiers and Borderlines in ManyParticle Physics, edited by R.A. Broglia and J.R. Schrieffer (North Holland, New York, 1988), p. 1.

[12] W.F. Brinkman and T.M. Rice, Phys. Rev. B 2, 4302 (1970); T. M. Rice and W. F. Brinkman, Phys. Rev. B 5, 4350 (1972).

[13] S. A. Shivashankar et al., J. Electrochem. Soc. 128, 2472 (1981); S. A. Shivashankar and J. M. Honig, Phys. Rev. B 28, 5695 (1983).

[14] S. A. Carter, T. F. Rosenbaum, P. Metcalf, J. M. Honig, and J. Spalek, Phys. Rev. Lett. 67, 3440 (1991); S. A. Carter, J. Yang, T.F. Rosenbaum, J. Spalek, and J.M. Honig, Phys. Rev. B 43, 607 (1991); S. A. Carter, Ph.D. thesis, University of Chicago, 1993; S. A. Carter, T.F. Rosenbaum, P. Metcalf, J. M. Honig, and J. Spalek, Phys. Rev. B 48, 16481 (1993). Our result that pressure and annealing produce a similar effect on the insulating phase is different from the findings of these authors in the metallic phase.

[15] S. Barker and J.P. Remeika, Solid State Commun. 8, 1521 (1970).

[16] J-H. Park and J. Allen, (to be published); J-H. Park, Ph.D. thesis, University of Michigan, 1993.

[17] G. A. Thomas, D.H. Rapkine, S.A. Carter, T.F. Rosenbaum, P. Metcalf, and J. M. Honig, J. Low Temp. Phys. (to be published).

[18] S. Shin, S. Suga, M. Taniguchi, M. Fujisawa, H. Kanzaki, A. Fujimori, H. Daimon, U. Ueda, K. Kosuge, and S. Kachi, Phys. Rev. B 41, 4993 (1990).

[19] Th. Pruschke, D. L. Cox, and M. Jarrell, Europhys. Lett. 21, 5 (1993); and Phys. Rev. B 47, 3553 (1993).

[20] G. Kotliar and M. J. Rozenberg (private communication).

[21] M. Takahashi, J. Phys. C 10, 1289 (1977), finds a kinetic energy ratio $1.10 D / U$; see also, A. H. MacDonald, S.M. Girvin, and D. Yoshioka, Phys. Rev. B 37, 9753 (1988), from which we obtain our energy ratio.

[22] R. M. Moon, Phys. Rev. Lett. 25, 527 (1970).

[23] W. Kohn, Phys. Rev. 133, A171 (1964).

[24] D. Baerswyl, C. Gros, and T. M. Rice, Phys. Rev. B 35, 8391 (1987).

[25] A. J. Millis and S. N. Coppersmith, Phys. Rev. B 42, 10807 (1990). 